Supporting Information for

\title{
Cold-Trap-Mediated Broad Dynamic Photodetection in Graphene-Organic Hybrid Photonic Barristors
}

\author{
Dong Un Lim, ${ }^{1}$ Sae Byeok Jo ${ }^{1,2, *}$ and Jeong Ho Cho ${ }^{1, *}$ \\ ${ }^{1}$ Department of Chemical and Biomolecular Engineering, Yonsei University, Seoul 03722 , \\ Korea. \\ ${ }^{2}$ Department of Materials Science and Engineering, University of Washington, Seattle, WA \\ 98195-2120, USA.
}
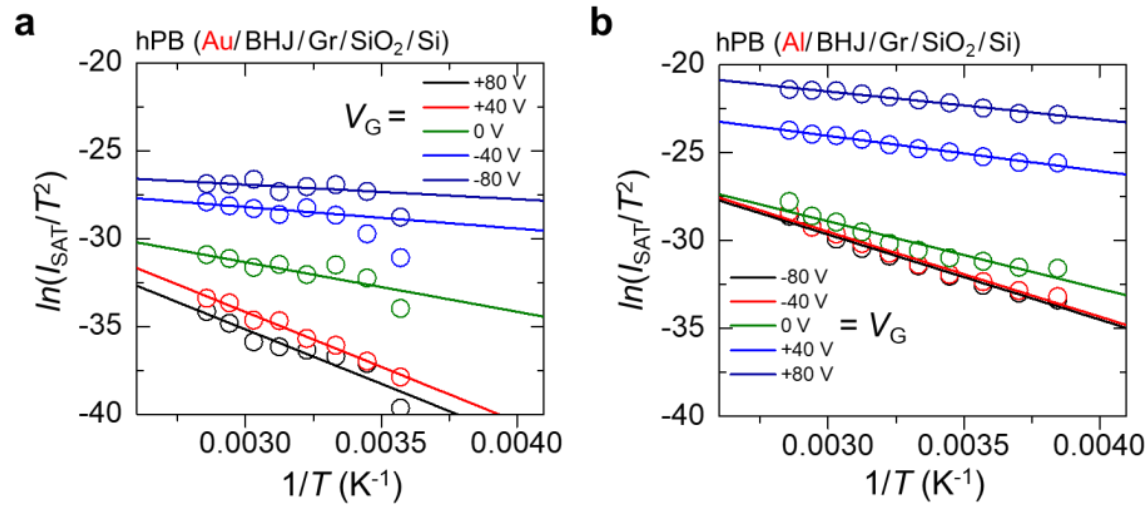

Fig. S1. Measurement of tunable injection barriers. $\ln \left(I_{\mathrm{D}} / T^{2}\right)$ versus $1 / T$ plots at various gate voltages for (a) $p$-channel and (b) $n$-channel hybrid photonic barristors. The charge injection at the graphene-organic interfaces was quantitatively investigated via examination of the temperature-dependent output characteristics in the temperature range of 190-350 K. The saturation current $\left(I_{\mathrm{SAT}}\right)$ under reverse bias can be described by the thermionic emission model as follows $(1)$

$$
I_{\mathrm{SAT}}=A A^{*} T^{2} e^{-\frac{q \varphi_{\mathrm{B}}}{k_{\mathrm{B}} T}}
$$

where $\varphi_{\mathrm{B}}$ is the SB height at the graphene-organic interface; $A$ is the area of the Schottky junction; $A^{*}$ is the effective Richardson constant; and $k_{\mathrm{B}}, T$, and $q$ are the Boltzmann constant, absolute temperature, and elementary charge, respectively. Then, we plotted $\ln \left(I_{\mathrm{SAT}} / T^{2}\right)$ versus $1 / T$ and calculated the SB height from the slope of each curve. The calculated SB heights and the $E_{\mathrm{F}}$ as functions of $V_{\mathrm{G}}$ are presented in Figure 2a in the main text. 
a
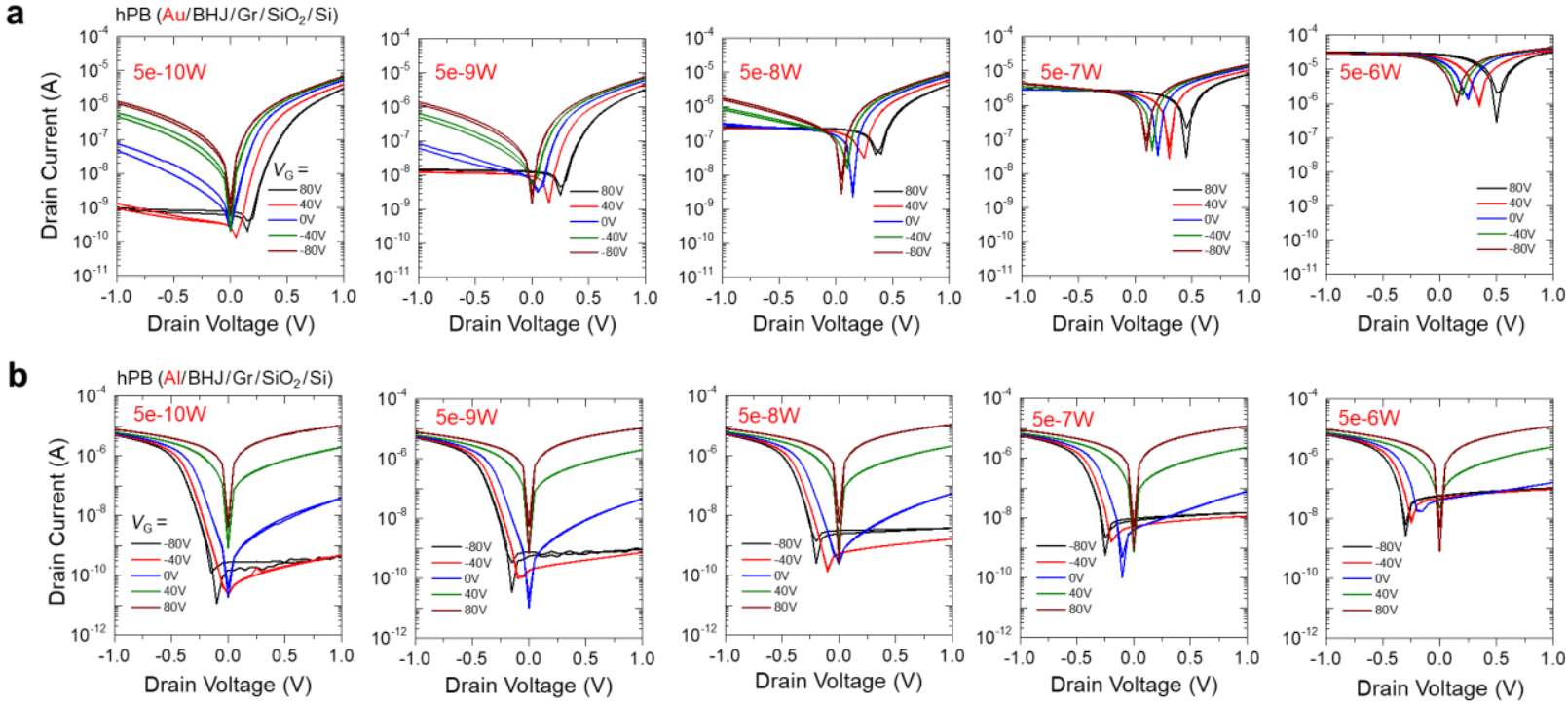

Fig. S2. Electrical characteristics of hPBs. Semi-logarithmic plots of $V_{\mathrm{G}}$-dependent output curves for hybrid photonic barristors with (a) Au top electrodes and (b) Al top electrodes under different illumination conditions
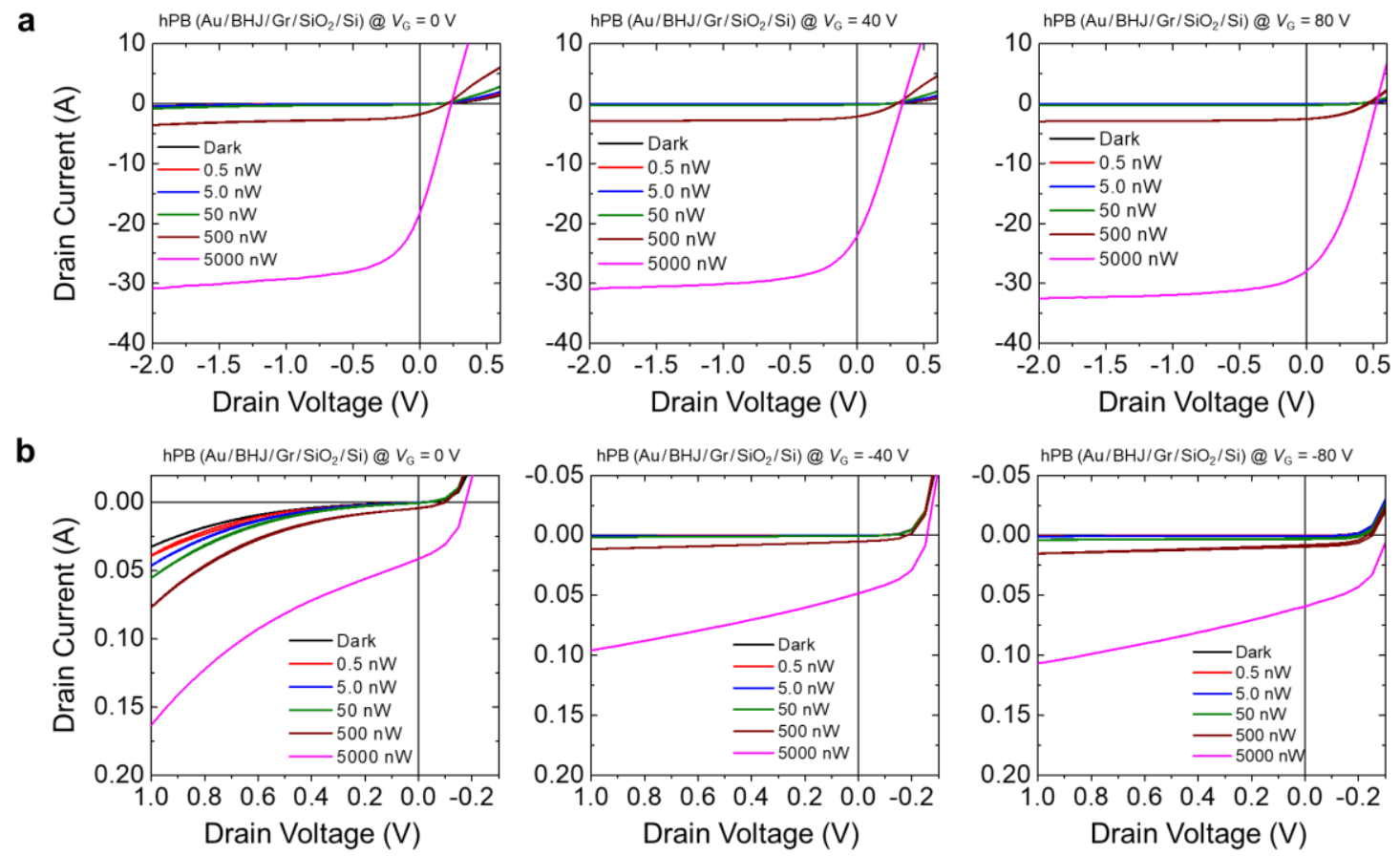

Fig. S3. Photovoltaic characteristics of hPBs. Linear plots of illumination-dependent output curves for hybrid photonic barristors with (a) Au top electrodes and (b) Al top electrodes under different gate voltages. 

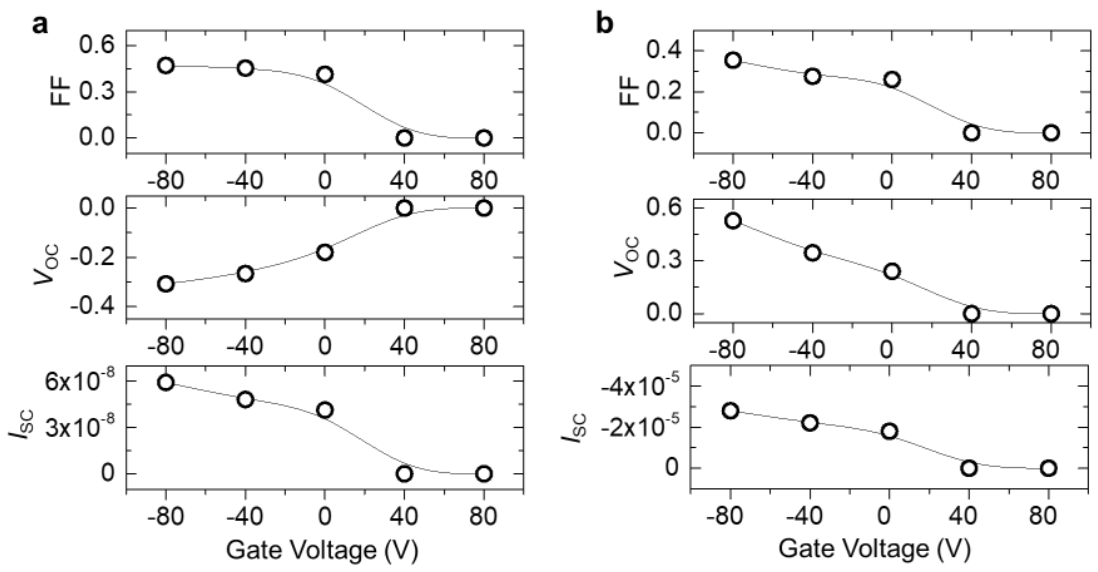

Fig. S4. Photovoltaic figure of merits of hPBs. Device parameters extracted from Figure S3 for hybrid photonic barristors with (a) Au top electrodes and (b) Al top electrodes. $F F$ : fill factor, $V_{\mathrm{OC}}$ : open-circuit voltage, $I_{\mathrm{SC}}$ : short-circuit current
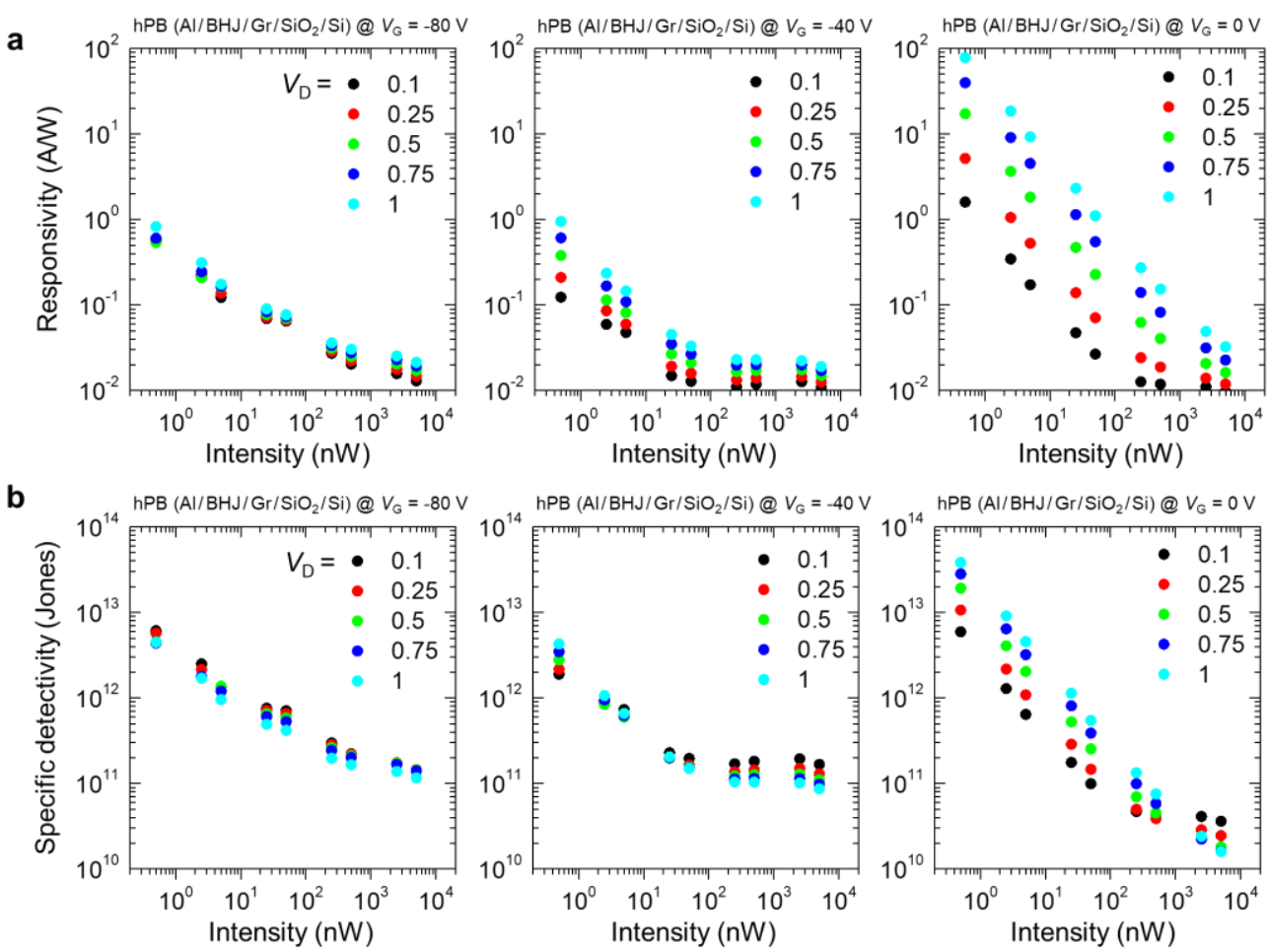

Fig. S5. Illumination-dependent electrical characteristics. Logarithmic plots of illumination-dependent (a) photoresponsivity and (b) specific detectivity in hybrid photonic barristors with $\mathrm{Al}$ top electrodes at different $V_{\mathrm{D}}$ and $V_{\mathrm{G}}$. 

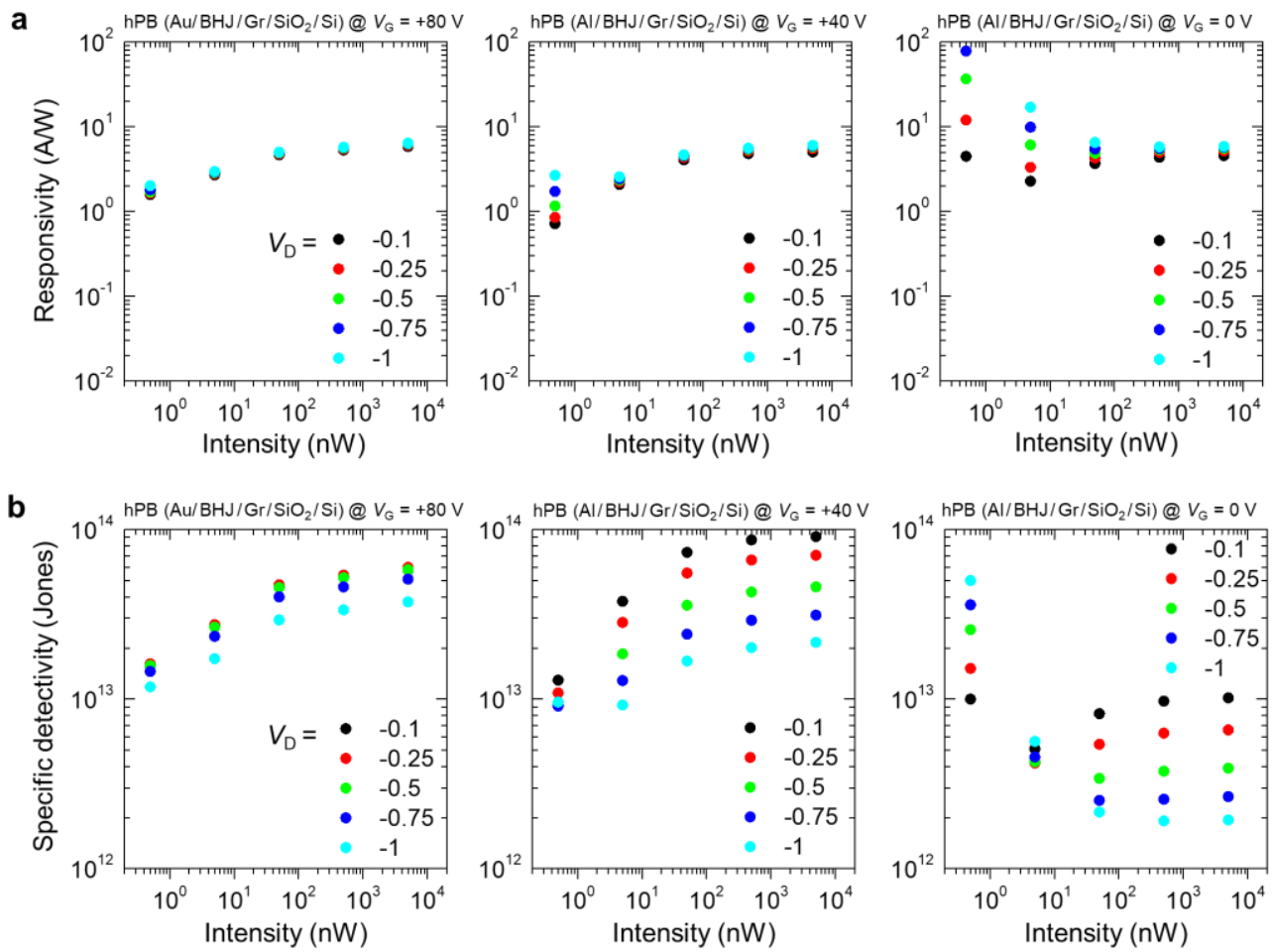

Fig. S6. Illumination-dependent electrical characteristics. Logarithmic plots of illumination-dependent (a) photoresponsivity and (b) specific detectivity in hybrid photonic barristors with $\mathrm{Au}$ top electrodes at different $V_{\mathrm{D}}$ and $V_{\mathrm{G}}$. 
a
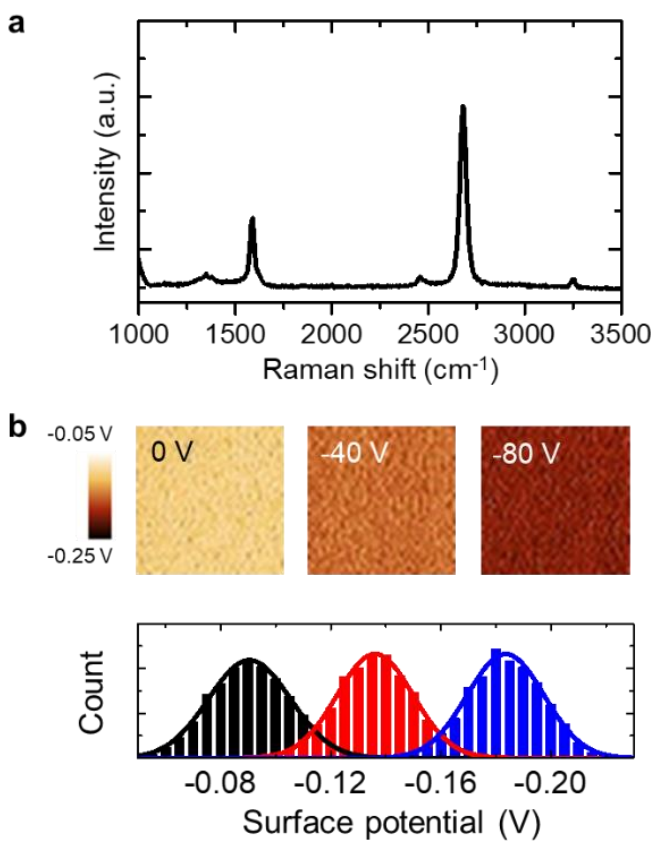

Fig. S7. (a) Raman spectrum of monolayer graphene layer. (b) Kelvin probe force microscopy (KPFM) images and the surface potential distribution of the graphene layer on a $\mathrm{SiO}_{2}$ gate dielectric $(300 \mathrm{~nm})$ under various $V_{\mathrm{G}}$. (c) Surface potential of the graphene/ $\mathrm{SiO}_{2}$ as a function of $V_{\mathrm{G}}$.

a Graphene $/ \mathrm{SiO}_{2}$
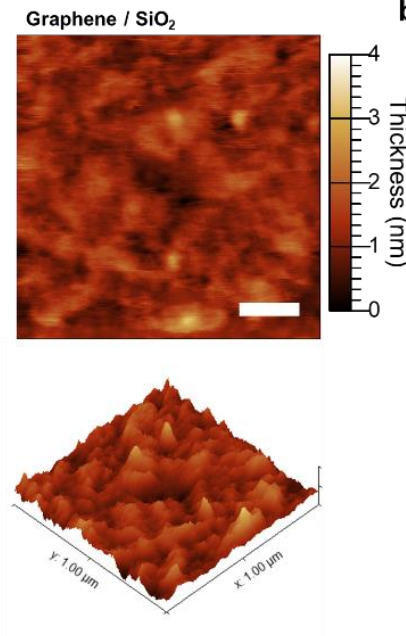

b PBDB-T:PC 71 BM / $\mathrm{SiO}_{2}$
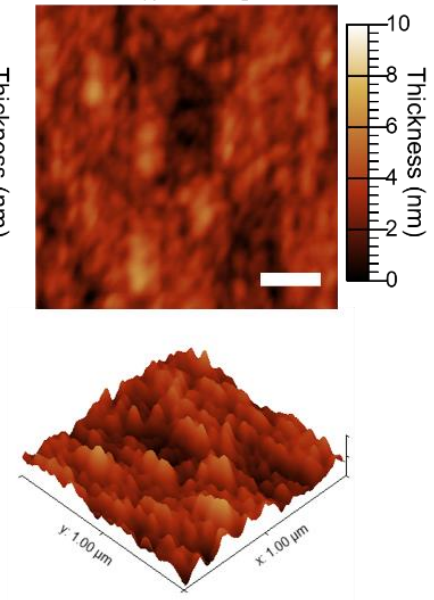

C PBDB-T:PC 71 BM / Graphene $/ \mathrm{SiO}_{2}$
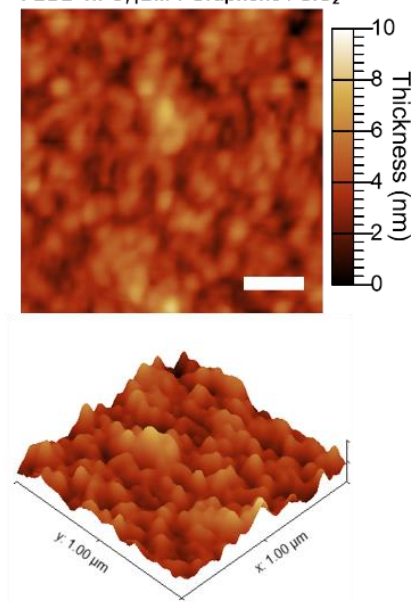

Fig. S8. The surface morphology of (a) graphene layer, (b) PBDB-T:PC $\mathrm{C}_{71} \mathrm{BM}$ layer on $\mathrm{SiO}_{2}$ and (c) PBDB-T:PC $71 \mathrm{BM}$ layer on graphene measured by the atomic force microscopy. The scale bar is $100 \mathrm{~nm}$ in length. 

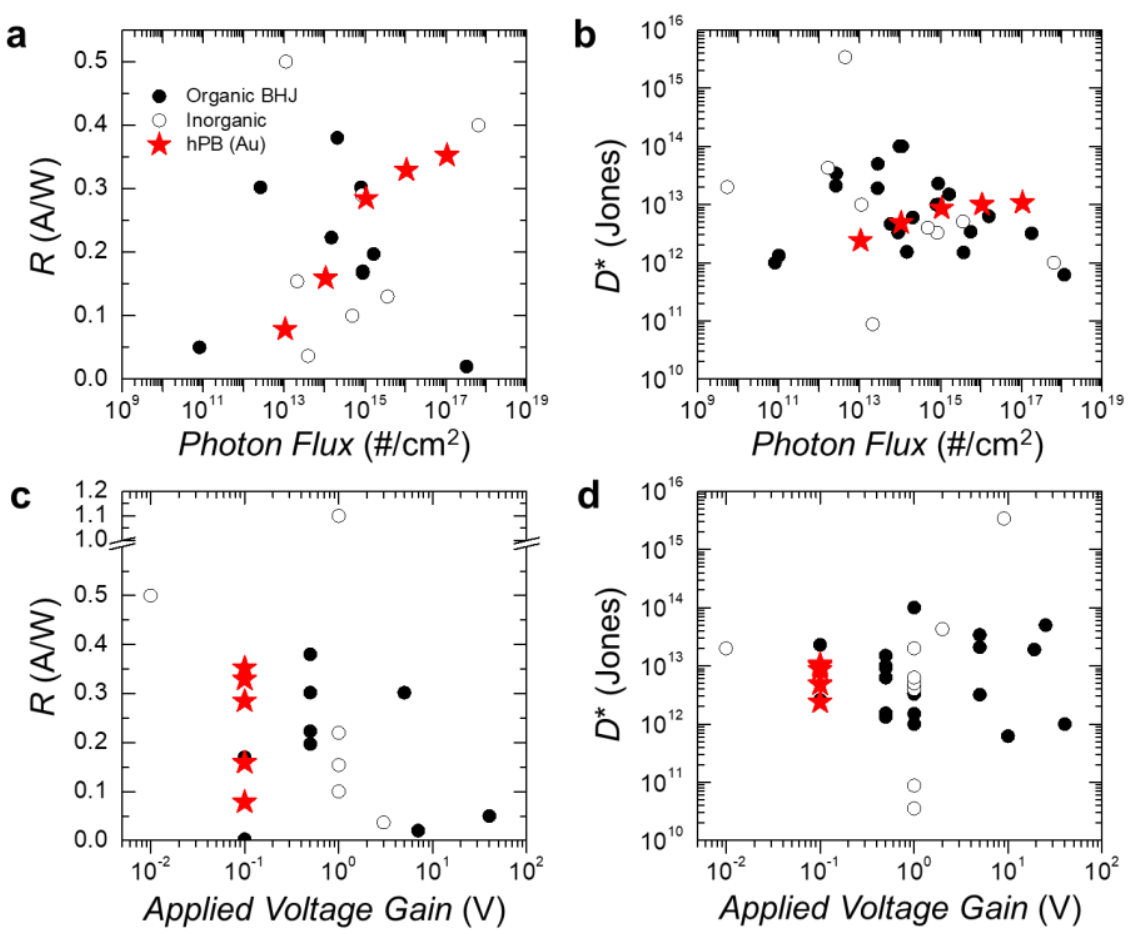

Fig. S9. Literature survey for photodiodes. Comparison of $(a, c)$ the photoresponsivity and $(b, d)$ specific detectivity depending on $(a, b)$ the photon flux and $(c, d)$ the applied voltage gain between the recently reported literatures and our work. Detailed parameters are included in Table S1 and S2. 
Table S1. Literature survey for organic PDs. Summary of device types, measurement conditions and the figure-of-merits in organic photodetectors.

\begin{tabular}{|c|c|c|c|c|c|c|c|c|c|}
\hline Type of Device & Material & $\begin{array}{l}V_{\mathbf{D}} \\
(\mathbf{V})\end{array}$ & $\begin{array}{c}\lambda \\
(\mathrm{nm})\end{array}$ & $\begin{array}{c}P_{\mathrm{IN}} \\
\left(\mathrm{mW} / \mathrm{cm}^{2}\right)\end{array}$ & $\begin{array}{c}R \\
(\mathrm{~mA} / \mathrm{W})\end{array}$ & $\begin{array}{c}D^{*} \\
\text { (Jones) }\end{array}$ & $\begin{array}{l}L D R \\
(\mathrm{~dB})\end{array}$ & $\begin{array}{c}\tau_{\text {rise }}(\mathrm{ms}) / \\
f_{-3 \mathrm{~dB}}(\mathrm{kHz})\end{array}$ & Ref \\
\hline Phototransistor & DPP/PCBM & -40 & 808 & $2.00 \mathrm{E}-05$ & $5.00 \mathrm{E}+01$ & $1.0 \mathrm{E}+12$ & - & $2.0 \mathrm{E}+01 /-$ & (2) \\
\hline Photodiode & PCDTBT/PC $71 \mathrm{BM}$ & -5 & 532 & $1.00 \mathrm{E}-03$ & - & $3.4 \mathrm{E}+13$ & - & $-/ 2.0 \mathrm{E}+00$ & (3) \\
\hline Photodiode & PCDTBT,DPP-DTT/PC ${ }_{71} \mathrm{BM}$ & 1 & 650 & - & $1.00 \mathrm{E}+02$ & $1.0 \mathrm{E}+12$ & 80.0 & $-/ 1.0 \mathrm{E}+02$ & (4) \\
\hline Photodiode & $\mathrm{PDDTT} / \mathrm{PC}_{61} \mathrm{BM}$ & -0.1 & 800 & $2.20 \mathrm{E}-01$ & $1.70 \mathrm{E}+02$ & $1.0 \mathrm{E}+13$ & 100.0 & $-1-$ & (5) \\
\hline Photodiode & $\mathrm{SQ} / \mathrm{PC}_{61} \mathrm{BM}$ & 1 & 700 & $1.60 \mathrm{E}+00$ & - & $3.4 \mathrm{E}+12$ & 30.0 & $5.0 \mathrm{E}-04 /-$ & (6) \\
\hline Photodiode & $\mathrm{P} 3 \mathrm{HT} / \mathrm{PC}_{61} \mathrm{BM}$ & 1 & 570 & $1.30 \mathrm{E}+00$ & - & $1.5 \mathrm{E}+12$ & - & $1.5 \mathrm{E}-02 / 5.6 \mathrm{E}+00$ & (7) \\
\hline Photodiode & $\mathrm{BPPC} / \mathrm{CuPc}$ & 7 & 650 & $1.00 \mathrm{E}+02$ & $2.00 \mathrm{E}+01$ & - & - & $2.0 \mathrm{E}-03 /-$ & (8) \\
\hline Photodiode (PM-type) & PBDTT-DPP/PC ${ }_{71} B M$ & 1 & 780 & $2.50 \mathrm{E}-02$ & $2.97 \mathrm{E}+04$ & $1.0 \mathrm{E}+14$ & 70.0 & $1.6 \mathrm{E}-01 /$ - & (9) \\
\hline Photodiode (PM-type) & PBDTT-ffQ ${ }_{x} / \mathrm{PC}_{61} \mathrm{BM}$ & 10 & 770 & $3.00 \mathrm{E}+02$ & $1.00 \mathrm{E}+03$ & $6.2 \mathrm{E}+11$ & - & $8.99 \mathrm{E}+01 /-$ & (10) \\
\hline Photodiode & PCPDTSBT/PCPDTSBT & -0.5 & 700 & $4.20 \mathrm{E}-02$ & $2.23 \mathrm{E}+02$ & $1.5 \mathrm{E}+12$ & 128.0 & $-/ 1.01 \mathrm{E}+01$ & (11) \\
\hline Photodiode & PCZ-Th-DPP & -1 & 650 & $1.80 \mathrm{E}-02$ & - & $4.6 \mathrm{E}+12$ & 109.0 & $4.53 \mathrm{E}-01 / 1.23 \mathrm{E}+00$ & (12) \\
\hline Photodiode & CllnPc/C60 & 1 & 705 & $2.54 \mathrm{E}-02$ & - & $3.3 \mathrm{E}+12$ & 77.2 & $-/ 2.85 \mathrm{E}-03$ & (13) \\
\hline Photodiode & PCE10:P3HT/PCBM & 0.5 & 780 & $2.57 \mathrm{E}-05$ & - & $1.3 \mathrm{E}+12$ & 76.5 & $-/ 1.0 \mathrm{E}-01$ & (14) \\
\hline Photodiode & P3HT & 0 & 520 & $7.89 \mathrm{E}-02$ & $3.80 \mathrm{E}+02$ & $6.0 \mathrm{E}+12$ & 201.0 & $2.69 \mathrm{E}-02 /-$ & (15) \\
\hline Photodiode & $\mathrm{PDPP} 3 \mathrm{~T} / \mathrm{PC}_{71} \mathrm{BM}$ & -0.5 & 820 & $4.00 \mathrm{E}-01$ & $1.97 \mathrm{E}+02$ & $1.5 \mathrm{E}+13$ & 104.0 & 4.3E-04 / 5.7E+03 & (16) \\
\hline Photodiode (PM-type) & $\mathrm{P} 3 \mathrm{HT} / \mathrm{PC}_{71} \mathrm{BM}$ & -5 & 650 & $5.50 \mathrm{E}+01$ & $2.00 \mathrm{E}+03$ & $3.2 \mathrm{E}+12$ & 118.0 & $-1-$ & (17) \\
\hline Photodiode (PM-type) & PTB7-Th/PC 61 BM & -19 & 625 & $8.87 \mathrm{E}-03$ & - & $1.91 \mathrm{E}+13$ & - & $1.0 \mathrm{E}+03 /-$ & (18) \\
\hline Photodiode (PM-type) & $\mathrm{P} 3 \mathrm{HT} / \mathrm{PC}_{61} \mathrm{BM}$ & -25 & 400 & $1.41 \mathrm{E}-02$ & $3.61 \mathrm{E}+05$ & $5.00 \mathrm{E}+13$ & - & $1.0 \mathrm{E}+03 /-$ & (19) \\
\hline Photoconductor & $\mathrm{PDPP} 3 \mathrm{~T} / \mathrm{PC}_{71} \mathrm{BM}$ & -0.5 & 680 & $4.60 \mathrm{E}+00$ & - & $6.30 \mathrm{E}+12$ & - & $2.7 \mathrm{E}+02 /-$ & (20) \\
\hline Photodiode & Zn-Porphyrin-dimer/PC ${ }_{61} \mathrm{BM}$ & 0 & 1130 & - & $3.28 \mathrm{E}+01$ & $8.80 \mathrm{E}+11$ & & $-1-$ & (21) \\
\hline Photodiode & Zn-Porphyrin-dimer/PC ${ }_{61} \mathrm{BM}$ & 0 & 1400 & - & $1.19 \mathrm{E}+02$ & $8.20 \mathrm{E}+10$ & & $-1-$ & (21) \\
\hline Photodiode & $\mathrm{P} 3 \mathrm{HT} / \mathrm{PC}_{61} \mathrm{BM}$ & -0.5 & 500 & $3.20 \mathrm{E}-01$ & $3.02 \mathrm{E}+02$ & $1.00 \mathrm{E}+13$ & 120.0 & $-1-$ & (22) \\
\hline Photodiode & p-DTS $\left(\mathrm{FBTTh}_{2}\right)_{2} / \mathrm{PC}_{7_{1} \mathrm{BM}}$ & -0.5 & 700 & - & $3.80 \mathrm{E}+02$ & $9.20 \mathrm{E}+12$ & 140.0 & $-/ 4.0 \mathrm{E}+03$ & (23) \\
\hline Photodiode & $1-\mathrm{BF} 4 / \mathrm{C}_{60}$ & 0 & 1440 & - & $1.30 \mathrm{E}-02$ & $3.70 \mathrm{E}+09$ & - & $-1-$ & (24) \\
\hline Photodiode & $1-\mathrm{TPFB} / \mathrm{C}_{60}$ & 0 & 1460 & - & $1.30 \mathrm{E}-02$ & $5.30 \mathrm{E}+10$ & - & $-1-$ & (24) \\
\hline
\end{tabular}




\begin{tabular}{lccccccccc} 
Photodiode & $2-\mathrm{BF} 4 / \mathrm{C}_{60}$ & 0 & 1590 & - & $1.80 \mathrm{E}-02$ & $7.00 \mathrm{E}+09$ & - & $-/-$ & $(24)$ \\
Photodiode & PDT/PC $61 \mathrm{BM}$ & -0.1 & 900 & - & $2.40 \mathrm{E}+00$ & $2.60 \mathrm{E}+12$ & - & $-/-$ & $(25)$ \\
Photodiode & PCDTBT-Imine/PC $71 \mathrm{BM}$ & 0 & 1200 & & $5.80 \mathrm{E}+01$ & $2.00 \mathrm{E}+11$ & 100.0 & $-/-$ & $(26)$ \\
\hline
\end{tabular}

Table S2. Literature survey for inorganic PDs. Summary of device types, measurement conditions and the figure-of-merits in inorganic photodetectors. The classification of the device type is explained in the main text.

\begin{tabular}{|c|c|c|c|c|c|c|c|c|c|}
\hline Type of Device & Material & $\begin{array}{l}V_{\mathbf{D}} \\
(\mathbf{V})\end{array}$ & $\begin{array}{c}\lambda \\
(\mathbf{n m})\end{array}$ & $\begin{array}{c}P_{\mathrm{IN}} \\
\left(\mathrm{mW} / \mathrm{cm}^{2}\right)\end{array}$ & $\begin{array}{c}\boldsymbol{R} \\
(\mathbf{m A} / \mathbf{W}) \\
\end{array}$ & $\begin{array}{c}D^{*} \\
\text { (Jones) }\end{array}$ & $\begin{array}{l}D D R \\
(\mathrm{~dB})\end{array}$ & $\begin{array}{r}\tau_{\text {rise }}(\mathrm{ms}) / \\
f_{-3 \mathrm{~dB}}(\mathrm{kHz})\end{array}$ & Ref \\
\hline Photoconductor & $\mathrm{CH}_{3} \mathrm{NH}_{3} \mathrm{PbI}_{3} / \mathrm{MoS}_{2}$ & 2 & 660 & $5.00 \mathrm{E}-04$ & $1.08 \mathrm{E}+04$ & $4.28 \mathrm{E}+13$ & - & $4.5 \mathrm{E}+01 /-$ & $(27)$ \\
\hline Photodiode & $\mathrm{In}_{0.79} \mathrm{Ga}_{0.21} \mathrm{As}_{0.45} \mathrm{P}_{0.55}$ & - & 1204 & - & $7.50 \mathrm{E}+02$ & - & - & $-/ 4.2 \mathrm{E}+07$ & $(28)$ \\
\hline Photoconductor & $\mathrm{CH}_{3} \mathrm{NH}_{3} \mathrm{PbI}_{3}$ & 3 & 780 & $1.00 \mathrm{E}-02$ & $3.67 \mathrm{E}+01$ & - & - & $2.0 \mathrm{E}+02 /-$ & $(29)$ \\
\hline Photoconductor & $\mathrm{CH}_{3} \mathrm{NH}_{3} \mathrm{PbI}_{3} / \mathrm{PDPP} 3 \mathrm{~T}$ & 1 & 835 & $5.00 \mathrm{E}-03$ & $1.54 \mathrm{E}+02$ & $8.80 \mathrm{E}+10$ & - & $-/-$ & $(30)$ \\
\hline Photodiode & $\mathrm{CH}_{3} \mathrm{NH}_{3} \mathrm{PbI}_{3}$ & 0 & 640 & $2.00 \mathrm{E}+02$ & $4.00 \mathrm{E}+02$ & $1.00 \mathrm{E}+12$ & 80.0 & $1.2 \mathrm{E}-03 /-$ & $(31)$ \\
\hline Photodiode & $\mathrm{CH}_{3} \mathrm{NH}_{3} \mathrm{PbI}_{3}$ & 0 & 740 & $3.00 \mathrm{E}-03$ & $5.00 \mathrm{E}+02$ & $1.00 \mathrm{E}+13$ & 112.0 & $5.8 \mathrm{E}-05 /-$ & $(32)$ \\
\hline Photodiode & $\mathrm{CH}_{3} \mathrm{NH}_{3} \mathrm{PbI}_{3}$ & 0 & 490 & $3.41 \mathrm{E}-01$ & $2.90 \mathrm{E}+02$ & $3.29 \mathrm{E}+12$ & - & $2.0 \mathrm{E}-02 /-$ & (33) \\
\hline Photodiode & $\mathrm{CH}_{3} \mathrm{NH}_{3} \mathrm{PbI}_{3}$ & 0 & 500 & - & $3.50 \mathrm{E}+02$ & $1.53 \mathrm{E}+11$ & - & $-1-$ & $(34)$ \\
\hline Photodiode & $\mathrm{CH}_{3} \mathrm{NH}_{3} \mathrm{PbI}_{3}$ & 0 & 650 & - & $4.00 \mathrm{E}+02$ & $3.00 \mathrm{E}+12$ & 95.0 & $-1-$ & $(35)$ \\
\hline Photodiode & $\mathrm{CH}_{3} \mathrm{NH}_{3} \mathrm{PbI}_{3} / \mathrm{PDPPTDTPT}$ & - & 900 & - & - & $1.00 \mathrm{E}+11$ & 95.0 & $-1-$ & $(36)$ \\
\hline Photodiode & $\mathrm{CH}_{3} \mathrm{NH}_{3} \mathrm{PbI}_{3}+\mathrm{PbS} \mathrm{QD}$ & 0 & 900 & $8.00 \mathrm{E}-01$ & $1.3 \mathrm{E}+02$ & $5.1 \mathrm{E}+12$ & 100.0 & $-1-$ & $(37)$ \\
\hline Photodiode & PbS QD & -1 & 975 & $1.00 \mathrm{E}-01$ & $1.00 \mathrm{E}+02$ & $4.00 \mathrm{E}+12$ & 60.0 & $1.0 \mathrm{E}-02 /-$ & $(38)$ \\
\hline Photoconductor & $\mathrm{CH}_{3} \mathrm{NH}_{3} \mathrm{PbI}_{3}+\mathrm{ZnO} \mathrm{NR}$ & -1 & 500 & - & $2.43 \mathrm{E}+04$ & $3.56 \mathrm{E}+10$ & - & $7.0 \mathrm{E}+02 /-$ & $(39)$ \\
\hline Photoconductor & $\mathrm{CH}_{3} \mathrm{NH}_{3} \mathrm{PbI}_{3}$ & 1 & 530 & $2.00 \mathrm{E}-06$ & $4.95 \mathrm{E}+03$ & $2.00 \mathrm{E}+13$ & 70.0 & $1.0 \mathrm{E}-01 /-$ & $(40)$ \\
\hline Photoconductor & $\mathrm{ZnO}$ nanoparticles & -9 & 700 & $1.25 \mathrm{E}-03$ & $1.001 \mathrm{E}+06$ & $3.40 \mathrm{E}+15$ & - & $2.5 \mathrm{E}-01 /-$ & $(41)$ \\
\hline Photodiode & Si (S1336-18BK) & -0.01 & 960 & & $5.00 \mathrm{E}+02$ & $2.00 \mathrm{E}+13$ & $\sim 120$ & $-1-$ & - \\
\hline Photodiode & InGaAs (G10899-030A) & -1 & 1550 & & $1.10 \mathrm{E}+03$ & $6.30 \mathrm{E}+12$ & $\sim 70$ & $-1-$ & - \\
\hline
\end{tabular}




\section{References}

1. H. Yang, J. Heo, S. Park, H. J. Song, D. H. Seo, K.-E. Byun, P. Kim, I. Yoo, H.-J. Chung, K. Kim, Graphene barristor, a triode device with a gate-controlled Schottky barrier. Science 336, 1140-1143 (2012).

2. H. Xu, J. Li, B. H. K. Leung, C. C. Y. Poon, B. S. Ong, Y. Zhang, N. Zhao, A high-sensitivity nearinfrared phototransistor based on an organic bulk heterojunction. Nanoscale 5, 11850-11855 (2013).

3. A. Pierre, I. Deckman, P. B. Lechêne, A. C. Arias, High Detectivity All-Printed Organic Photodiodes. Advanced Materials 27, 6411-6417 (2015).

4. A. Armin, R. D. Jansen-van Vuuren, N. Kopidakis, P. L. Burn, P. Meredith, Narrowband light detection via internal quantum efficiency manipulation of organic photodiodes. Nature Communications 6, 6343 (2015).

5. X. Gong, M. Tong, Y. Xia, W. Cai, J. S. Moon, Y. Cao, G. Yu, C.-L. Shieh, B. Nilsson, A. J. Heeger, High-Detectivity Polymer Photodetectors with Spectral Response from $300 \mathrm{~nm}$ to $1450 \mathrm{~nm}$. Science 325, 1665-1667 (2009).

6. M. Binda, A. Iacchetti, D. Natali, L. Beverina, M. Sassi, M. Sampietro, High detectivity squaraine-based near infrared photodetector with nA/cm2 dark current. Applied Physics Letters 98, 073303 (2011).

7. G. Azzellino, A. Grimoldi, M. Binda, M. Caironi, D. Natali, M. Sampietro, Fully Inkjet-Printed Organic Photodetectors with High Quantum Yield. Advanced Materials 25, 6829-6833 (2013).

8. T. Morimune, H. Kajii, Y. Ohmori, Photoresponse Properties of a High-Speed Organic Photodetector Based on Copper-Phthalocyanine Under Red Light Illumination. IEEE Photonics Technology Letters 18, 2662-2664 (2006).

9. R. Nie, X. Deng, L. Feng, G. Hu, Y. Wang, G. Yu, J. Xu, Highly Sensitive and Broadband Organic Photodetectors with Fast Speed Gain and Large Linear Dynamic Range at Low Forward Bias. Small 13, 1603260 (2017).

10. S. Tong, J. Yuan, C. Zhang, C. Wang, B. Liu, J. Shen, H. Xia, Y. Zou, H. Xie, J. Sun, S. Xiao, J. He, Y. Gao, J. Yang, Large-scale roll-to-roll printed, flexible and stable organic bulk heterojunction photodetector. npj Flexible Electronics 2, 7 (2018).

11. S. Yoon, H. S. Ryu, J. U. Ha, M. Kang, T. L. Nguyen, H. Y. Woo, D. S. Chung, High-Performance Near-Infrared-Selective Thin Film Organic Photodiode Based on a Molecular Approach Targeted to Ideal Semiconductor Junctions. The Journal of Physical Chemistry Letters 10, 5647-5653 (2019).

12. S. Z. Hassan, H. J. Cheon, C. Choi, S. Yoon, M. Kang, J. Cho, Y. H. Jang, S.-K. Kwon, D. S. Chung, Y.-H. Kim, Molecular Engineering of a Donor-Acceptor Polymer To Realize Single Band Absorption toward a Red-Selective Thin-Film Organic Photodiode. ACS Applied Materials \& Interfaces 11, 2810628114 (2019).

13. C. W. Joo, J. Kim, J. Moon, K. M. Lee, J.-E. Pi, S.-Y. Kang, S.-D. Ahn, Y.-S. Park, D. S. Chung, Highperformance fab-compatible processed near-infrared organic thin-film photodiode with $3.3 \times 1012$ Jones detectivity and 80\% external quantum efficiency. Organic Electronics 70, 101-106 (2019).

14. J. Kim, S. Yoon, K. M. Sim, D. S. Chung, Rational design of a junction structure to realize an NIRselective narrowband organic thin-film photodiode. Journal of Materials Chemistry C 7, 4770-4777 (2019).

15. M. Kang, S. Yoon, J. Cho, J. Kim, D. S. Chung, Reactive Dedoping of Polymer Semiconductors To Boost Self-Powered Schottky Diode Performances. ACS Applied Materials \& Interfaces 11, 8365-8373 (2019). 
16. X. Zhou, D. Yang, D. Ma, Extremely Low Dark Current, High Responsivity, All-Polymer Photodetectors with Spectral Response from $300 \mathrm{~nm}$ to $1000 \mathrm{~nm}$. Advanced Optical Materials 3, 15701576 (2015).

17. Z. Zhao, C. Li, L. Shen, X. Zhang, F. Zhang, Photomultiplication type organic photodetectors based on electron tunneling injection. Nanoscale 12, 1091-1099 (2020).

18. L. Li, F. Zhang, W. Wang, Q. An, J. Wang, Q. Sun, M. Zhang, Trap-Assisted Photomultiplication Polymer Photodetectors Obtaining an External Quantum Efficiency of 37 500\%. ACS Applied Materials \& Interfaces 7, 5890-5897 (2015).

19. L. Li, F. Zhang, W. Wang, Y. Fang, J. Huang, Revealing the working mechanism of polymer photodetectors with ultra-high external quantum efficiency. Physical Chemistry Chemical Physics 17, 30712-30720 (2015).

20. X. Zhou, D. Yang, D. Ma, A. Vadim, T. Ahamad, S. M. Alshehri, Ultrahigh Gain Polymer Photodetectors with Spectral Response from UV to Near-Infrared Using ZnO Nanoparticles as Anode Interfacial Layer. Advanced Functional Materials 26, 6619-6626 (2016).

21. J. D. Zimmerman, E. K. Yu, V. V. Diev, K. Hanson, M. E. Thompson, S. R. Forrest, Use of additives in porphyrin-tape/C60 near-infrared photodetectors. Organic Electronics 12, 869-873 (2011).

22. X. Liu, H. Wang, T. Yang, W. Zhang, X. Gong, Solution-Processed Ultrasensitive Polymer Photodetectors with High External Quantum Efficiency and Detectivity. ACS Applied Materials \& Interfaces 4, 3701-3705 (2012).

23. I. K. Kim, B. N. Pal, M. Ullah, P. L. Burn, S.-C. Lo, P. Meredith, E. B. Namdas, High-Performance, Solution-Processed Non-polymeric Organic Photodiodes. Advanced Optical Materials 3, $50-56$ (2015).

24. M. Young, J. Suddard-Bangsund, T. J. Patrick, N. Pajares, C. J. Traverse, M. C. Barr, S. Y. Lunt, R. R. Lunt, Organic Heptamethine Salts for Photovoltaics and Detectors with Near-Infrared Photoresponse up to $1600 \mathrm{~nm}$. Advanced Optical Materials 4, 1028-1033 (2016).

25. J. Han, J. Qi, X. Zheng, Y. Wang, L. Hu, C. Guo, Y. Wang, Y. Li, D. Ma, W. Qiao, Z. Y. Wang, Lowbandgap donor-acceptor polymers for photodetectors with photoresponsivity from $300 \mathrm{~nm}$ to $1600 \mathrm{~nm}$. Journal of Materials Chemistry C 5, 159-165 (2017).

26. A. E. London, L. Huang, B. A. Zhang, M. B. Oviedo, J. Tropp, W. Yao, Z. Wu, B. M. Wong, T. N. Ng, J. D. Azoulay, Donor-acceptor polymers with tunable infrared photoresponse. Polymer Chemistry 8 , 2922-2930 (2017).

27. Z.-Y. Peng, J.-L. Xu, J.-Y. Zhang, X. Gao, S.-D. Wang, Solution-Processed High-Performance Hybrid Photodetectors Enhanced by Perovskite/MoS2 Bulk Heterojunction. Advanced Materials Interfaces 5 , 1800505 (2018).

28. X. Fengnian, J. K. Thomson, M. R. Gokhale, P. V. Studenkov, W. Jian, W. Lin, S. R. Forrest, An asymmetric twin-waveguide high-bandwidth photodiode using a lateral taper coupler. IEEE Photonics Technology Letters 13, 845-847 (2001).

29. X. Hu, X. Zhang, L. Liang, J. Bao, S. Li, W. Yang, Y. Xie, High-Performance Flexible Broadband Photodetector Based on Organolead Halide Perovskite. Advanced Functional Materials 24, 7373-7380 (2014).

30. S. Chen, C. Teng, M. Zhang, Y. Li, D. Xie, G. Shi, A Flexible UV-Vis-NIR Photodetector based on a Perovskite/Conjugated-Polymer Composite. Advanced Materials 28, 5969-5974 (2016).

31. B. R. Sutherland, A. K. Johnston, A. H. Ip, J. Xu, V. Adinolfi, P. Kanjanaboos, E. H. Sargent, Sensitive, Fast, and Stable Perovskite Photodetectors Exploiting Interface Engineering. ACS Photonics 2, 11171123 (2015). 
32. H. L. Zhu, J. Cheng, D. Zhang, C. Liang, C. J. Reckmeier, H. Huang, A. L. Rogach, W. C. H. Choy, Room-Temperature Solution-Processed NiOx:PbI2 Nanocomposite Structures for Realizing HighPerformance Perovskite Photodetectors. ACS Nano 10, 6808-6815 (2016).

33. D. Li, G. Dong, W. Li, L. Wang, High performance organic-inorganic perovskite-optocoupler based on low-voltage and fast response perovskite compound photodetector. Scientific Reports 5, 7902 (2015).

34. L. Salamandra, N. Y. Nia, M. Di Natali, C. Fazolo, S. Maiello, L. La Notte, G. Susanna, A. Pizzoleo, F. Matteocci, L. Cinà, L. Mattiello, F. Brunetti, A. Di Carlo, A. Reale, Perovskite photo-detectors (PVSKPDs) for visible light communication. Organic Electronics 69, 220-226 (2019).

35. Y. Wang, D. Yang, X. Zhou, S. M. Alshehri, T. Ahamad, A. Vadim, D. Ma, Vapour-assisted multifunctional perovskite thin films for solar cells and photodetectors. Journal of Materials Chemistry C 4, 7415-7419 (2016).

36. L. Shen, Y. Lin, C. Bao, Y. Bai, Y. Deng, M. Wang, T. Li, Y. Lu, A. Gruverman, W. Li, J. Huang, Integration of perovskite and polymer photoactive layers to produce ultrafast response, ultraviolet-tonear-infrared, sensitive photodetectors. Materials Horizons 4, 242-248 (2017).

37. C. Liu, K. Wang, P. Du, E. Wang, X. Gong, A. J. Heeger, Ultrasensitive solution-processed broad-band photodetectors using $\mathrm{CH} 3 \mathrm{NH} 3 \mathrm{PbI} 3$ perovskite hybrids and $\mathrm{PbS}$ quantum dots as light harvesters. Nanoscale 7, 16460-16469 (2015).

38. F. P. García de Arquer, X. Gong, R. P. Sabatini, M. Liu, G.-H. Kim, B. R. Sutherland, O. Voznyy, J. Xu, Y. Pang, S. Hoogland, D. Sinton, E. Sargent, Field-emission from quantum-dot-in-perovskite solids. Nature Communications 8, 14757 (2017).

39. J. Yu, X. Chen, Y. Wang, H. Zhou, M. Xue, Y. Xu, Z. Li, C. Ye, J. Zhang, P. A. van Aken, P. D. Lund, $\mathrm{H}$. Wang, A high-performance self-powered broadband photodetector based on a CH3NH3PbI3 perovskite/ZnO nanorod array heterostructure. Journal of Materials Chemistry C 4, 7302-7308 (2016).

40. L. Gao, K. Zeng, J. Guo, C. Ge, J. Du, Y. Zhao, C. Chen, H. Deng, Y. He, H. Song, G. Niu, J. Tang, Passivated Single-Crystalline CH3NH3PbI3 Nanowire Photodetector with High Detectivity and Polarization Sensitivity. Nano Letters 16, 7446-7454 (2016).

41. F. Guo, B. Yang, Y. Yuan, Z. Xiao, Q. Dong, Y. Bi, J. Huang, A nanocomposite ultraviolet photodetector based on interfacial trap-controlled charge injection. Nature Nanotechnology 7, 798-802 (2012). 